\title{
FRAMEWORK FOR MONITORING FIREWALL FUNCTIONALITY USING INTRUSION DETECTION SYSTEMS
}

\author{
Peter Kiprono Kemei \\ Department of Computer \\ Science, Egerton University \\ Njoro, Kenya.
}

\author{
William P.K. Korir \\ Department of Computer \\ Science, Egerton University \\ Njoro, Kenya
}

\author{
Joseph Mbugua Chahira \\ Department of IT \\ Nkabune Technical Training \\ Meru, Kenya
}

\begin{abstract}
In the last few years, the intranet and Internet has experienced explosive growth due to number of benefits. Internet is insecure which makes security of private networks system an imported limitation. Firewall is installed as the first step of securing private networks. Firewalls are implemented at the block point of private network to protect them from external attacks through restricted defined rules and policies reaching network interface. Regular complaints have been raised due to invasion, intrusions and attacks of private networks even with the presence of firewalls. For purpose of confirmation, real time framework needs to be implemented to observe, examine effectiveness and functionality of firewalls by installing Network Intrusion Detection Systems (NIDS) security software within network perimeter to examine firewall operation. NIDS detects, offensive, inaccurate, or irregular action on a network and they are proper for any types of institute for defending the networks and systems. By setting up framework according to defined rules and policies deviation are reported automatically where administrator can check the events examined or audit to check if the firewall complies according to configured rules or policies where some are complex and high-level to implement all rules setup. The reported events enable the administrator to enforce and implement the appropriate rule which make the network safer to use.
\end{abstract}

Keywords: Firewall, Networks, Intrusions, Detection, Systems, Framework.

\section{INTRODUCTION}

Intrusions and attacks are the main threats against networks and information security. With rapidly growing illicit activities in networks, intrusion detection systems as a component of defense- in-depth are very necessary because traditional firewall techniques cannot provide complete protection against intrusion [12]. NIDS have become an essential component of computer security to detect these attacks before they inflict widespread damage [13]. They are used to monitor the usage of such systems and to detect the apparition of insecure states. They detect attempts and active misuse by valid users of the information systems or external parties to abuse their privileges or exploit security vulnerabilities [8]. NIDS make a robust application for identify, recognized and response from security violations, it needs the framework that cooperates with connected and related several components for accurate, intelligent adaptive and extensible with composite to an integrated system [6]. The main aim for integrating NIDS with a firewall includes filtering, management of update data set, the sensor can take dissimilar actions based on how they are configured and event reaction process. Security policies are decisive step to secure exacting system since it identifies the security properties. There are strong confirmation that the installation of up to date NIDS system that are position at the perimeter can defer significant protection for networks [5] which supplement major shortcomings of firewall.

\section{RELATED WORK}

Firewalls utilize static, manually configured, security policies to differentiate genuine traffic from non-genuine traffic. They prevent illegal external users from accessing computing resources on the internal network, avoid the negative untrusted relations impact of a break in, provide a reliable connection to the internet where users do not implement their own insecure private connections and control internal user access to the outside network to prevent the export information. Firewalls cannot provide complete protection against some attacks and intrusion [12]. They have shortcoming such as inability to prevent networks from interior attacks [11]. They may not be properly configured to stop all apprehensive packets based on rules or policies due to complex and expertise of unknown traffic or emerging threats. Utilize manually configured set of rules to differentiate genuine traffic from permitted traffic. Firewalls cannot protect against attacks that bypass the rules and policies implemented. Interior system may have dial-out ability to join to an internet service providers. An internal local area network may support a modem band that provides dial-in capability for mobile employees and teleworks which pose network security threats [2], [7], [17], [20] and cannot protect against the transfer of malicious programs or files. Firewalls are essential part of network security, but they do not provide airtight perimeter protection, due to highlighted shortcomings. In order to be sure of firewall functionality installation of updated NIDSs inserted within network environs to supplement shortcomings and examine firewall functionality could be viable. Network administrators can perform a more secure network system by using NIDSs as an extra layer of protection beside the firewall. Protecting information system today must be done in a layered process, which includes technology and user intervention. NIDSs have software potential of identifying illegal use, misuse and exploitation of computer by attackers and intruders [15].NIDS are intended to identity suspicious and wicked activities that tend to compromise the 
confidentiality, integrity and guarantee of network computer systems [10]. Unlike firewalls that filter "bad traffics", NIDS analyzes packets to detect apprehensive traffic packets attempts. From the survey report CSI 2012, NIDS was ranked seventh with $62.4 \%$ [14] as per usage by network administrator to improved network security. The number and severity of these attacks has been increasing continuously [9]. NIDSs automate examine and evaluate the attacks [16] and used to classify asses and report permits network activities so that correct actions can be implemented to prevent supplementary damage [1]. NIDSs detection techniques join tools or a method that collect and audits the information from any number of sources, after collection it evaluate the information and determines problems existing in packets at some stage in transmission. It identifies and reports unauthorized or malicious network action. The main goals of NIDSs are to detect intrusions that have occurred or that are in the process of occurring in attempting to understand or moderate suspicious activities [4]. NIDS are submissive device that simply detects problems and cause alarms or alerts the security administrators. Detects the patterns of known attacks by corresponding pattern with the rule base. It can recognize the signatures of malware programs and the types of attacks. Encryption can be severe setback for networkbased NIDS because it cannot handle encrypted network traffic [3],[18]. The encrypted traffic should be ignored by NIDS for high performance and to reduce false positives. NIDS decodes SSL and TLS traffic and stops inspection of the encrypted data. Only the SSL handshakes of each connection are inspected to determine that the last client-side handshake packet was not crafted to evade the NIDS. Once the data determined to be encrypted, further inspections of the data on the connection are stopped [19]. Detects variation from regular actions of network systems by implementing protocol and traffic anomaly detection. It detects abnormal behavior, such as extraordinary increase in traffic from a port, protocol, timestamp and several uninterrupted ineffective attempts at logging into the computer and network.

\section{OBJECTIVES, APPROACH AND CHARACTERISTICS}

NIDS are proactive technique used to prevent attacks from entering the network by examining various data record and detection deportment of pattern gratitude sensor when an attack are identified, intrusion prevention block and log the aberrant data. The main objective of proposed framework is to provide early caveat from intrusion security violation with knowledge based, dynamic, smart in classifying and distinguish of packet data, if curious or mischievous are detected, alert triggers and event response execute. The mechanism trigger allow process packet data associated with the event. NIDS objective is to examine stream network traffic detecting distinguishes and recognized any packets that could trace any security breaches. The proposed methodical approach differs from previous since the concepts examining firewall operation using NIDS approach in detecting normal usages and malicious activities using diverse data which leads to improvement and enhances mechanism with combine anomaly misuse based and event parameters data input. The methodological approach improvement mechanism which uses data from sources. The parameters data input includes different structure, label, variable of data detected, collection from public DNS registry, public IP Block list, universal resource locator blacklist, NIDS snort rules, vulnerability from common vulnerability and exposures, data pattern from bastion host and DMZ, signature, dynamic update patch, Log events server, web applications, firewall and network environment, spam, IP Block list, virus definition, policies definition, event from NIDS and regular reported IP address or hosts. The basic idea of exploratory firewall operation using NIDS makes a strong system for identify, recognized and reaction from security breaches, the framework connects and related several component for perfect, intelligent, adaptive and extensible components composite to an integrated system. The characteristics of the proposed framework consist of:-

- Filtering. It involves data collection from initiating dataset formerly, after effectively pass from filtering and screening. In the process, filtering, screening and proxy with firewall function, such as IP Address, port number used, protocol used and timestamp. The propose IP tables under NIDS transmission and sorting packet with accordance to security policy set. Firewalls provide diverse rule logic with dissimilar parameters based on rule set.

- Administration inform to control dataset consist of signature recognition, rules, policy, pattern, process attack, URL blacklist, renew patch, log system, listing variant of virus and normal expression, all these collected and labelled to classify attack patterns .This technique depends on the input in sequence collected in a database. The sequence in the database come from a diversity of information collected and stored periodically. In some cases, emerging attacks based on preceding patterns, particularly the attacks from malicious threat, on acquaintance process, execute composite and coalesce the data residing on the database to be sorted, queries and reused as input. The learning process occurs to unite and choose quickly by evaluating robust of the data in the database for analysis in preventing unknown attacks of intrusion.

- Sensor detects the packet events found on how they are configured. If threat evaluation passes, the system triggers event reply with status alarm or risk rating status. If an alert triggers, then the alert fused with other existing alert to decrease the number of alert with the same cause. Risk level is the quantitative measure of a network's suspicious threat level before event response alleviation. When new events are detected and sensor detect an attack, an analyst can check to see if the event's regular activity components, store in archive event database if not in list. Database component gets rate mark and lists it within risk rating can deeper examination with signature corresponding and behaviour scrutiny.

- The event reply are group into reactive response are trigger and implement after intrusion have been detected and proactive reply, aimed to anticipate actions to prevent an anticipated attack, By using this approach every unknown activity or doubtful threat has labelling according to NIDS rule based on priority classification which used in validating the framework based on information traced in order to make sound decisions. 
4. FRAMEWORK CONCEPTUAL MODEL

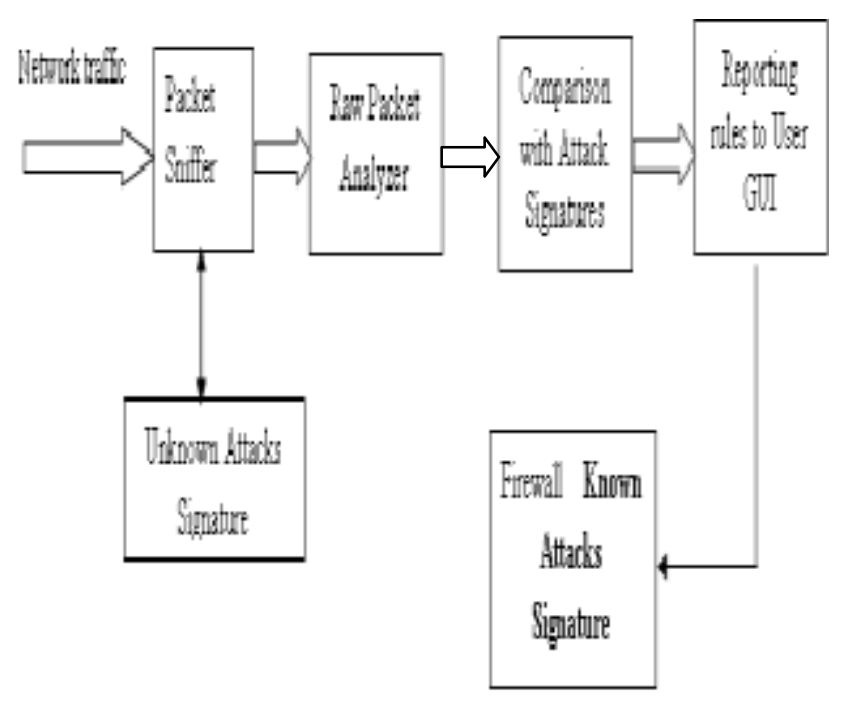

Figure 1: Framework Conceptual Model

From the figure 1 packet sniffer module captures all incoming and outgoing network traffic. The packet sniffer installed at the edges of the network traces all suspicious packets since it operates in promiscuous mode. In raw packet analyzer module identifies attack packet signature based on packet header of particular attack identification packets details such as follows by source and destination IP address, ports, protocols, header size, Time to Live, flag bits used. Attacks identification involves extraction of essential information traces packets details and compare with raw packet analyzer to determine module actual attack launched. Reporting attack details module involves reporting the attack to the participate for decisions making such as rules, actions events, state of network, reports and alerts. It involves the conceptual model framework for examining firewall operation using NIDS main aims at identifying unknown suspicious packets both private and untrusted network to trace the firewall rule targeted or affected informs the administrator in making sound decisions. Specification of attack details such as source victim IP addresses, time stamp of attack and type of firewall rule target.

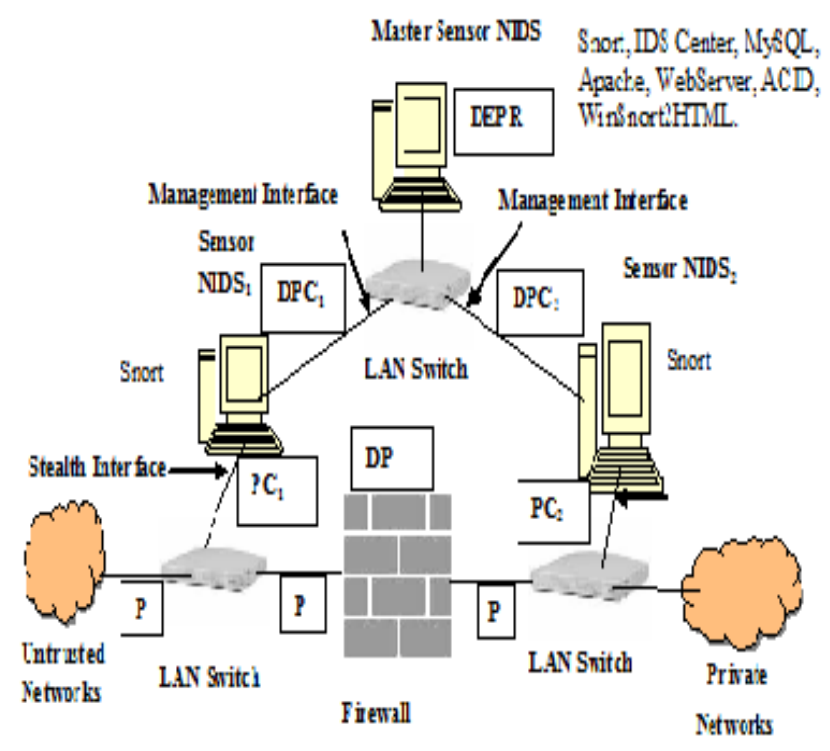

Figure 2: Framework Main Components and Network Traffic Flow Lifecycle

From figure 2 packets can either originate from private or untrusted network. If the packet $[\mathrm{P}]$ originates from an untrusted network it first encounters LAN switch. The packet [P] flows to the firewall where its main is to filter traffic depending on the rule-set configured. If the packets are drop then the packet lifecycle ends. If the Packet has some suspicious packets the sensor NIDS $_{1}$ have the ability to detect and a packet copy $\left[\mathrm{PC}_{1}\right]$ is created by LAN switch capabilities. The packets are delivered through stealth interface sent detected packet copy $\left[\mathrm{DPC}_{1}\right]$ for examination and analysis according to rules and policies set in Master Sensor NIDS as detected examined packet result [DEPR]. If the firewall allows, the detected packet [DP] encounters a second LAN switch where again a packet copy $\mathrm{PC}_{2}$ created if any suspicion detected packet copy $\left[\mathrm{DPC}_{2}\right]$ should be sent to Master sensor NIDS for examination and analysis according to rules and policies defined in Master sensor NIDS to confirm if firewall truly enforce the configured rules and policies. It's normal packet then it passes to the private networks, the packet reaches the destination and packet ends the life cycle. If the packet originates from the private networks then similar procedure takes place as packet originating from untrusted networks.

\section{FRAMEWORK IMPLEMENTATION MAIN COMPONENTS}

\subsection{Framework Implementation Monitoring Proposed Algorithm}




\begin{tabular}{|c|c|c|c|c|}
\hline \multirow{6}{*}{$\begin{array}{l}\text { Incoming } \\
\text { traffic }\end{array}$} & & \multicolumn{2}{|c|}{ IF } & \multirow{2}{*}{$\begin{array}{c}\text { THEN } \\
\text { CHECK POINT } \\
\text { FIREWALL }\end{array}$} \\
\hline & & SENSOR NIDS $_{1}$ & SENSOR NIDS $_{2}$ & \\
\hline & \multirow[b]{2}{*}{ Drop } & Alert & No Alert & Normal Operation \\
\hline & & Alert & Alert & PROBLEM \\
\hline & \multirow[b]{2}{*}{ Accept } & Alert & Alert & Normal Operation \\
\hline & & Alert & No Alert & PROBLEM \\
\hline \multirow{4}{*}{$\begin{array}{l}\text { Outgoing } \\
\text { traffic }\end{array}$} & \multirow[b]{2}{*}{ Drop } & No Alert & Alert & Normal operation \\
\hline & & Alert & Alert & PROBLEM \\
\hline & \multirow[b]{2}{*}{ Accept } & Alert & Alert & Normal Operation \\
\hline & & No Alert & Alert & PROBLEM \\
\hline
\end{tabular}

Table 1: Framework Monitoring Algorithm

The framework implementation examining algorithm main goal is to detect whenever there is network suspicious traffic then framework ability is to reveal the problem inclusive with captured traffic details as shown in table1.The goal of installing the sensor NIDS $_{1}$ in untrusted networks before the check point firewall and sensor NIDS $_{2}$ after the check point firewall in private networks to examine the traffic before filtration and after passing the check point firewall. The goals achieved are:-

1. Examine if check point firewall enforces configured rules /policies of incoming and outgoing traffic;

2. Examining of attacks or intrusion originating from private networks and confirmation if check point firewall enforces configured rules/policies;

3. Examining of successful packets filtered by check point firewall from private/untrusted network;

4. Gives administrators room to analyse types of attacks, intrusions and adjust the security rules / policies accordingly.

5. Adoptable as one of the source of computer, network and data communication forensic investigation.

\subsection{Framework Implementation Model}

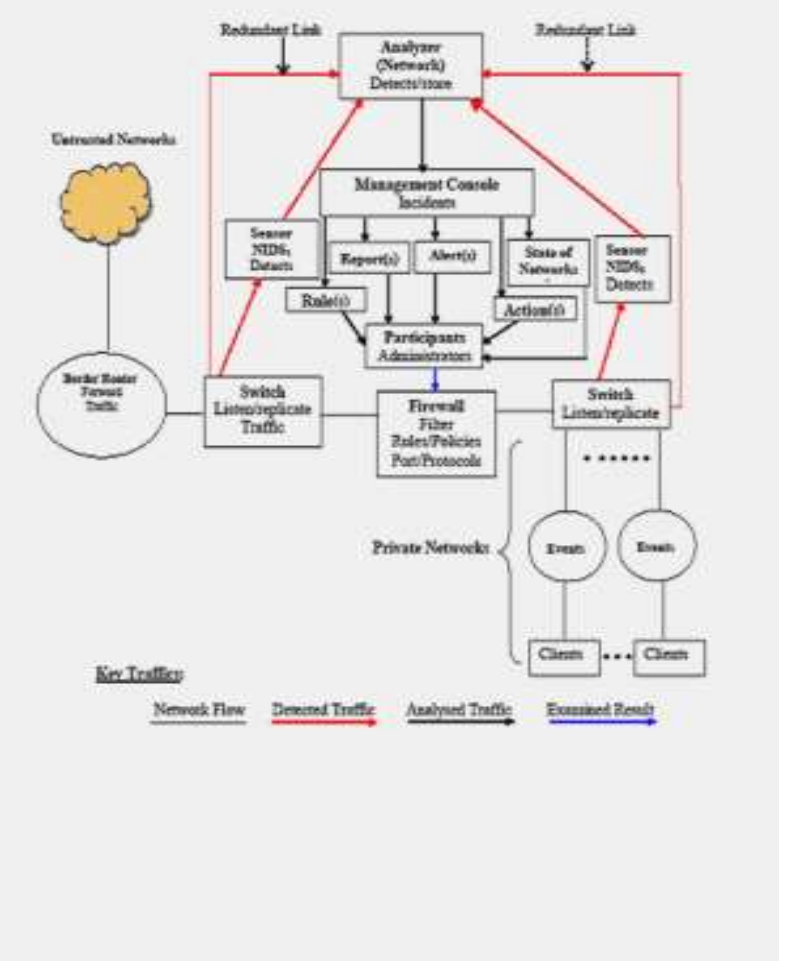

Figure 3: Framework Implementation Model

From figure 3 framework implementation model have advantages over the existing model since most of implemented models trace the packet flow only once during transit in terms of examining firewall operation between private network and firewall, untrusted network, firewall and network in general. Framework implementation model checks network traffic flows by determined by the module attributes. Framework implementation model checks network traffic flows by determined by the module attributes capabilities. It involves identification of the packets definition breaking, malfunction rules/policies affected in normal operation of firewall or network in general. The module attributes play their roles based on the functionalities. The network packet first encounters the LAN switch which have listen and replicates packets on transit and pass to the firewall to filter according to rule and policies configured. The Sensor NIDSs installed at network perimeter uses in-built abilities to detect suspicious packets flows according to signatures, pattern and bahaviour of the packet then packet captured pass to analyzer network determine breach rules or policies. The analyzed traffic passes to management console for identification of specific type of incidents. The participant implement the decisions according to the examined results which enables networks administrators to define, configured appropriate rules/policies to encounters firewall and network problems in general. The two redundant links supplement the link between LAN switch, Sensor NIDS and network analyzer in case link failure the link connects automatically making the examination and detection of network traffic continuously without any interruption. 


\section{FRAMEWORK IMPLEMENTATION MODEL RESULTS AND DISCUSSION}

Most firewalls are administered by network administrator which sometimes may be complex to examine it operation depend on the nature of network. The framework proves to examine firewall operation based on configured rules-set and detects network problems or attacks from the tests and experiment analyzed.

\subsection{Absence of Internal Filtering}

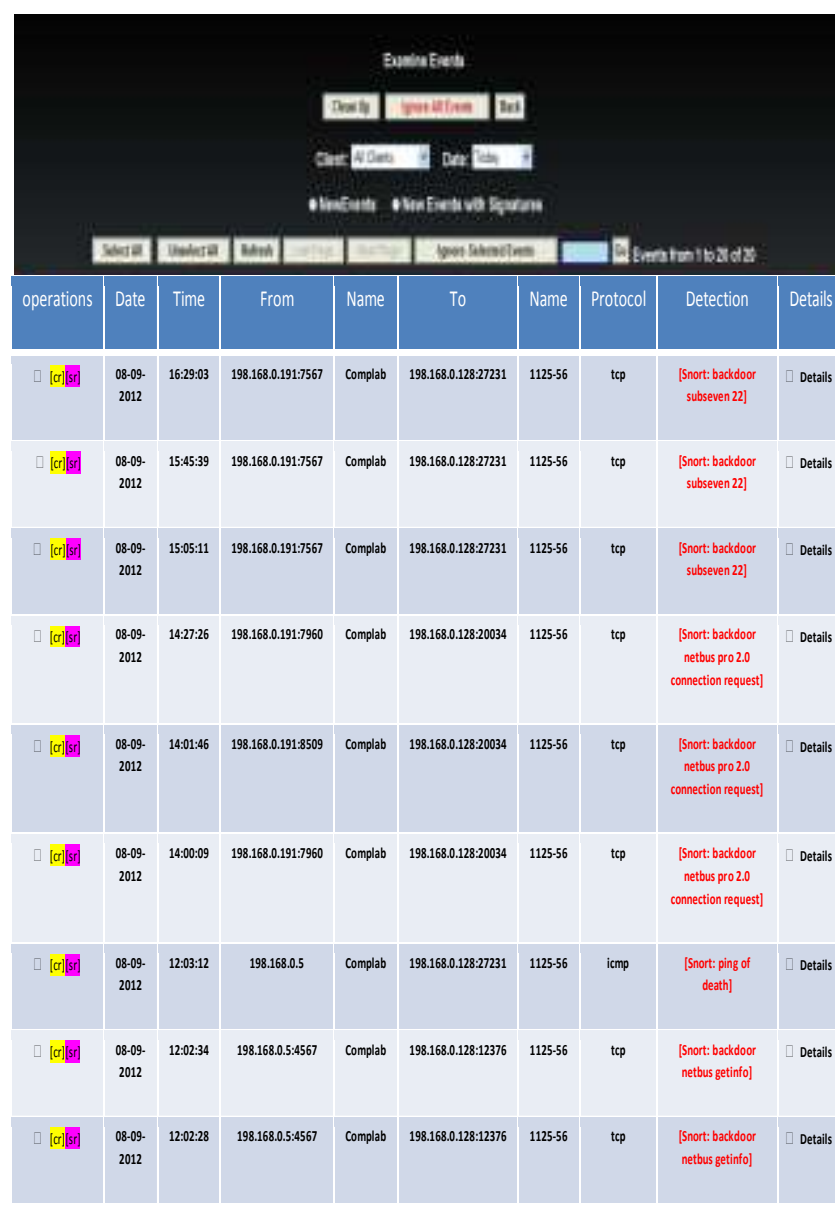

Figure 4: Framework Detection of Absence of Internal Filtering

From figure 4 the framework revealed that firewall was able to act according to configured rule-sets based on known traffic but cannot filter unknown traffics and its details internally. Once a host connected to internal LAN it can send packets to any host across network without internal filtering by the firewall. Experiments were conducted to test the framework by sending traffic both physical LAN and untrusted networks such wireless. All traffic got to framework indicating that no firewall filtering for unknown traffic across network boundaries which pose networks threats and intrusion. The framework was able to detect the traffics and full packets descriptions in details which can enable network administrator to implement the necessary steps on the configuration of firewall based on framework detected reports.

\subsection{Heavy Traffic on Specific Ports and Protocols}

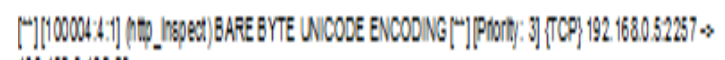
192180.1880

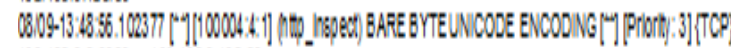

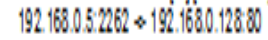

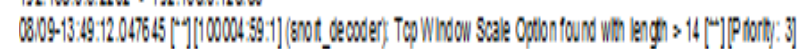

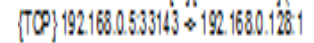

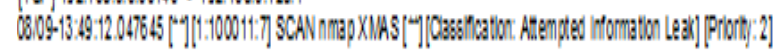

TQ] $1221605314 * 132160.121$

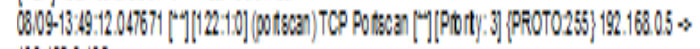

192160.128

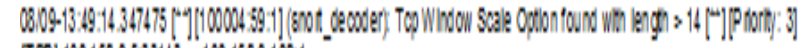

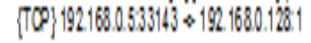

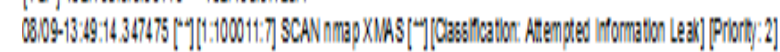

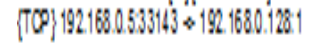

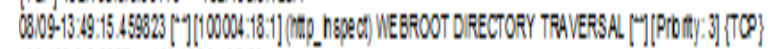

$192180.4277 \times 192680.1280$

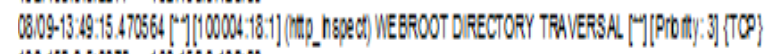

$1221805278 \times 131680.1280$

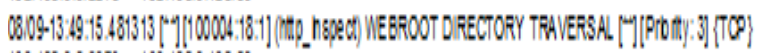

$192160527 \% \div 181680.128 \%$

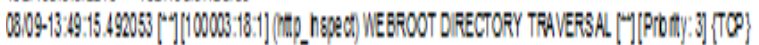

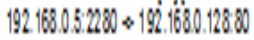

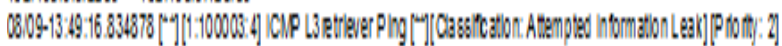

[OPP $192160.5 \times 19218018$

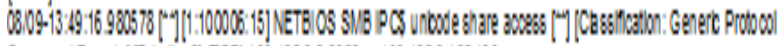

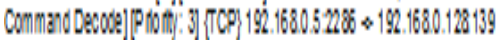

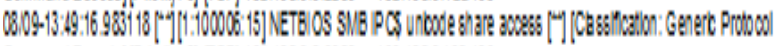

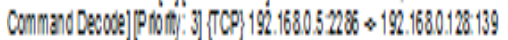

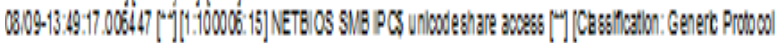

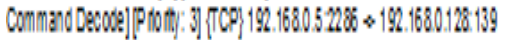

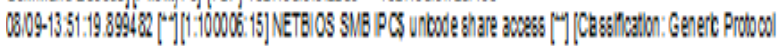

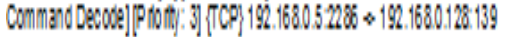

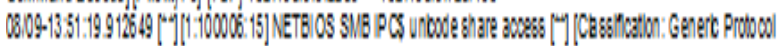

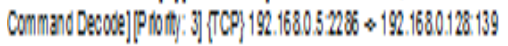

Figure 5: Heavy Traffic on Specific Ports and Protocols

From figure 5 the reported events with high number of regular traffic on ports $135,137,138,139,80,23,8080,8180$ and 445 as per the tests and experiment captured by the framework. Port 135 normally used to remotely managed service including DHPC server, DNS server detected by framework as among reported events using TCP protocol. Ports 137 used for NetBIOS-ns (name service), 138 used for NetBIOS-dgm (datagram service) and 139 used for NetBIOSssn (session service) are all network services used by NetBIOS LAN hosts for communication among themselves detected by the framework as the among most examined reported traffic using TCP protocols. Ports 80 which were initial block and open for specific services specific ACK and SYN flags but the framework also detected heavy traffic on the same port. On further analysis it revealed that the port reported used flag FIN, URG and PUSH which initial was not block on firewall rule-set chain policy utilizing TCP protocol. Framework detected heavy traffic detected on port 23 used for remote access using TCP specifically ICMP telnet protocol for unencrypted text communications, initial UDP protocol was block using port 23 in firewall chain policy. Ports 8080 and 8081 uses TCP protocols especially HTTP alternate (HTTP_alt). Port 8080 commonly used by Web proxy and caching, APACHE servers was also detected by framework as among heavy traffic examined events among port not initially configured on the firewall chain rule policy. This 
implies that the framework was able to detect and report the port and classifies the type of protocol used and other packet details. Port 445 using TCP protocols which used Server Massage Block (SMB) and Inter Process Communication (\$IPC) for files sharing on Microsoft active directory. This was detected by framework as among the port with heavy traffic examined events reported. All these ports and protocols provide essential information about the status of the hosts within the network. This information could be used to map network services and launch network attacks or intrusion if firewall rule sets and policies are not fully implemented and operational as expected.

\subsection{Suspicious Packets and Internal IP Addresses}

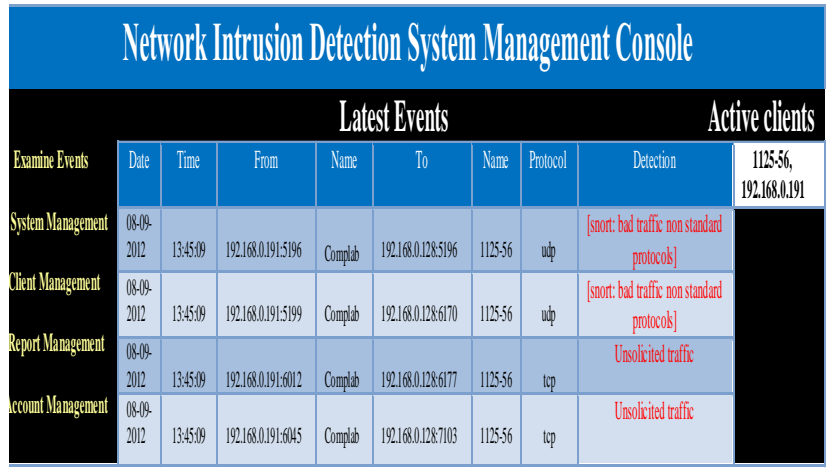

Figure 6: Suspicious Packets and Internal IP Addresses

From figure 6 the tested results from the framework traced numerous suspicious packets and internal IP address which firewall could not filter especially for network which are not centrally managed, where IP addresses are not assigned dynamically specific ports and protocols could be filtered. The framework detected bad traffic non- standard IP protocols, unsolicited connection mostly using TCP using port 445 and UDP using port 111 respectively were the most reported suspicious packets with specific hosts IP addresses source names and their destinations. Other detected packets and logged events revealed evidence by the framework was mis-configured software and hosts on the network. These two ports detected as bad traffic non standard IP protocol which portmapper to access network services both internally and externally due undetected traffic by firewall. After resetting the firewall rule set then the firewall filtered the traffic.

\subsection{Suspicious Foreign Packets and IP Addresses}

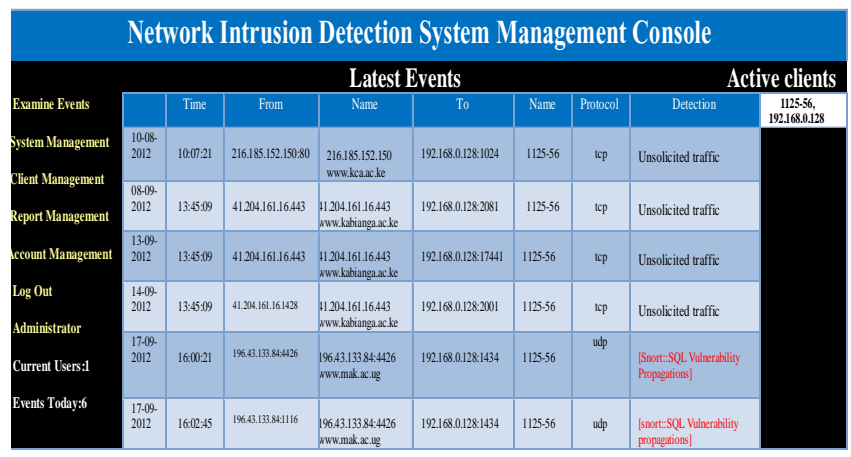

Figure 7: Suspicious Foreign Packets and IP Addresses
From figure 7 the framework detected suspicious foreign packets and IP addresses even with the installation of firewall expected to provide high degree of protection. After testing the framework using internal network foreign IP address, the IP address was delivery to the destination and the framework could detect these suspicious foreign and IP address. This indicates that the firewall was mis-configured since the number and frequency of packets on the network from foreign IP addresses and the times at which they were highly reported by the framework. Although some of the packets were drop but the fact that some packets were detected by the framework which indeed reveals a significant firewall security flaws. The framework detected TCP or UDP packets originating from untrusted network and submitted to port 1434 which were propagated by the vulnerabilities in Microsoft SQL server database management system which could launch a denial of service attacks against internet hosts and show slow down network speed by engaging the bandwidth. Many suspicious source address report by framework associated with attempting to connect to port 1024, a port that if often used by backdoor application which includes Netspy, port 10000 which host Webmin and port 161 which is associated with SNMP services.

\section{CONCLUSIONS AND FURTHER WORK}

With new emerging network threats and mostly firewall are normally configured manually they cannot be examined to critically review normally operation which leads to the option of developing a framework to examine firewall operation and network in general. Since there exist programs which have capability to detected and examine every packet flow within network setup they can utilized and implemented to examine firewall operation. This detected software includes NIDS specifically snort software which is open source having mechanism to inspect packet signature patterns and behaviours patterns. It can be utilized within network perimeters purposely to examine firewall operation and network in generally.

Firewall as network component vital in connecting two homogeneous networks. The operation of firewall has not been clearly examined to check its operation. These lead to conceptual of developing a framework which purposed installed within network environment to examined firewall operation. The framework can be adaptable since it functions on real time, which implies that it is active, persistent and careful consideration of any detected network anomalies based on framework information which forms foundation of knowledge in the light of detection grounds that support firewall and network weaknesses before making conclusion. The aim of the framework is to examine firewall and explores an issue of concern, a triggered detected packet which breaches network security policies configured in the firewall and engage to explore an order which lead to new understanding and appreciation information before implementing corrective measure. Firewall and other networks protection systems do not see packets patterns nor do they report on events that do break their rules where attacks and intruders may that advantage to exploit the network services without being noticed. The proposed framework can provide extended information on possible incidents management where administrator and users can use in providing defense in depth analysis of firewall operation and current network security status. Firewall do not see what happen within themselves where approve request are not saved or traced. Pattern attacks are detected by the framework 
instantly and problems of firewall as well. Generated events and reports provides a solid base for any network incident, valuable information directly from the source, long run or incidental problems as well which enable network administrator to analysis and make sound decision based on reported events. By setting up framework according to rules and policies deviation are reported automatically where administrator can check the events examined or audit to check if the firewall is compliant according to configured rules or policies where some are complex and high-level. The reported events enable the administrator to enforce and implement the appropriate rule which make the network safer to use. The framework application as integrated part of the network information technology landscapes any new application or internet both from internal or untrusted networks is check from day one instantly. Further research should be carried for cases of IP spoofing events, watch list IP assigning methods, detected attacks which generates false positives alerts from NIDSs and over reported detected network traffic which should improve the framework performance.

\section{REFERENCES}

[1]. Abdelhalim, .. T. K. N. A., 2010. IDS Adaptation for an Efficient Detection in High Speed Networks. s.l., IEEE Conference on Internet Monitoring and Protection..

[2]. Brian, K. R. B. W., 2010. Firewalls for Dummies. 2nd ,ISBN: 0-7645-4048-3. ed. s.1.:s.n.

[3]. Carl, .. E. S. J. M., 2004. Intrusion Detection \& Prevention. ISBN: 0072229543 ed. s.l.:s.n.

[4]. Carter, E., 2010. Intrusion Detection Systems. 1st ed. Indianapolis, Indiana: Cisco Press.

[5]. Craig, V., 2009. SCADA Forensics with snort IDS, s.1.: ECU publication, (2009)

[6]. Deris, A. M., 2011. Pitcher Flow: Unified Integration for Intrusion Prevention System. singapore, IACSIT press.

[7]. Gouda, M. A. X. L., 2008. A Model of Stateful Firewalls and its Properties. IEEE International Conference on Dependable Systems and Networks., IV(10), pp. 1-15.

[8]. Herve, M. D. a. A. W., 1999. Towards a taxonomy of intrusion-detection systems. Ruschlikon, Switzerland, Computer Networks Elsevier.

[9]. Indraneel, M. M. C. \&. S. C., 2011. A Comparative Study of Related Technologies of Intrusion Detection \& Prevention Systems. Information Security, 2(4), pp. 512-526.

[10].Juniper , 2008. Juniper networks. [Online] Available at: http://www.juniper.net/us/en/productsservices/security/netscreen/ns5200/[Accessed , August 2012].

[11].Katkar, D. S. B. a. V., 2010. Tolerant Distributed Intrusion Detection System using Packet Filter Firewall and State Transition Tables. International Journal of Computer Applications (0975 - 8887), Volume 8No.11(Novel Architecture for Intrusion), pp. pg 29-32.

[12].Kobayashi, Y. B. a. H., 2003. Iintrusion detection systems: Technology and Development. IEEE
Computer Society Press.. Nihon Univesity and Beihang University, IEEE Computer Society Press.

[13].Richard, e. '., 2000. Evaluating Intrusion Detection Systems. Wood Street, Lexington, IEEE ComputerSociety Press.

[14]. Robert, R., 2011. Computer Crime and Security Survey, s.1.: Computer Security Institute.

[15].Saira Beg, e. a., 2010. Feasibility of Intrusion Detection System with High Performance Computing. International Journal for Advances in Computer Science, 1(1), pp. 1-14.

[16].Scarfone, K. .. M. P., 2010. Guide to intrusion detection and prevention systems (IDPS), chicago: NIST Special Publication.

[17].Sheth, C. T. R., 2011. Performance Evaluation and Comparative Analysis of Network Firewalls. IEEE International Conference on Devices and Communications (ICDeCom)., III(9), pp. 1-21.

[18].Skrobanek, P., 2011. Intrusion Detection Systems.. ISBN 978-953-307-167-1 ed. s.1.:s.n.

[19].Toprak, M., 2009. Intrusion Detection System Alert Correlation With Operating System Level Logs,, s.l.: İzmir Institute of Technology.

[20].Wes Noonan, I. D., 2010. Firewall Fundamentals. ISBN: 1-58705-221-0. ed. s.1.:s.n. 